\title{
A fluorine turns a medicinal benzodiazepine into NPS: the case of flualprazolam
}

\author{
Panagoula-Stamatina A. Ntoupa ${ }^{1} \cdot$ Ioannis I. Papoutsis ${ }^{1}$ (D) Artemisia A. Dona ${ }^{1} \cdot$ Chara A. Spiliopoulou ${ }^{1}$. \\ Sotiris A. Athanaselis ${ }^{1}$
}

Received: 23 October 2020 / Accepted: 10 December 2020 / Published online: 2 February 2021

(c) The Author(s) 2021

\begin{abstract}
Purpose The purpose of this review is to summarize the existing knowledge on flualprazolam, a novel 'designer' benzodiazepine that derives from the fluorination of the phenyl moiety in the ortho-position of alprazolam.

Methods An extensive literature search was carried out in PubMed, Google Scholar and World Wide Web using relevant keywords. All articles found were gathered, and the available information is presented.

Results This article reviews the existing knowledge on chemistry, pharmacology, toxicology, prevalence and current legal status of flualprazolam. Moreover, forensic and clinical cases where flualprazolam was involved worldwide, as well as flualprazolam seizures, along with the methods for its determination in biological samples are presented.

Conclusions The recent flualprazolam-related cases have raised concerns to regulatory authorities and international stakeholders suggesting that flualprazolam should be under international control. The urgent international control of flualprazolam, despite the limited information on clinical effects and pharmacologic characteristics available, is an important measure for the prevention of its increasing abuse worldwide.
\end{abstract}

Keywords Flualprazolam · Designer benzodiazepines · New psychoactive substances (NPS) · Toxicity · Drug addiction · Legal status

\section{Introduction}

The current widespread use of novel psychoactive substances (NPS) results in a significant number of intoxications or deaths, the investigation of which is a challenging issue for the forensic laboratories. NPS with similar structures to controlled substances enter rapidly the illicit drug arena, and until they are scheduled, they are widely distributed and used legally. Benzodiazepines are frequently prescribed drugs that are also misused by drug users as a substitute or aiming to hinder the negative effects or withdrawal symptoms of opiates. The list of illegal benzodiazepine derivatives has increased in the last 10 years, as the chemical substitution of typical benzodiazepines or their metabolites has led to a

Ioannis I. Papoutsis

ipapoutsis@med.uoa.gr

1 Department of Forensic Medicine and Toxicology, School of Medicine, National and Kapodistrian University of Athens, 75, Mikras Asias Street, Goudi, 11527 Athens, Greece large number of new substances that lack clinical safety or approved clinical uses [1].

From January 2019 to April 2020, 10 NPS benzodiazepines were identified in toxicology cases reported to United Nations Office on Drugs and Crime (UNODC), with flualprazolam, etizolam and flubromazolam accounting for $64 \%$ of all identified NPS [2]. Flualprazolam is a novel 'designer' benzodiazepine that has received much attention in the last 3 years. It is a fluorinated analogue of alprazolam, and a benzodiazepine that exhibits central nervous system (CNS) depressive effects [3]. Although its synthesis was patented in as early as 1976 [4], flualprazolam was never marketed as a medicinal drug. In 2017, the first flualprazolam use for illegal purpose was reported [5]. Since then, an expanding number of flualprazolam seizures have been reported. Flualprazolam is sold mainly as counterfeit tablets or in combination with other psychoactive substances [6]. It can be abused in numerous ways and is often taken in conjunction with other illicit drugs to increase their effects, to counteract the effect of opioid withdrawal or to ameliorate the aftereffects of stimulants use [7]. Its extensive recreational use by drug 
addicts has resulted in a significant number of flualprazolam intoxications, lethal or not. Flualprazolam has been detected during the investigation of postmortem, clinical or driving under the influence of drugs (DUID) cases, and this fact has raised concerns to international stakeholders suggesting that flualprazolam should be under international control [8].

The present paper is an overview of all available information on chemistry, pharmacology, toxicology, prevalence and current legal status of flualprazolam. Cases where flualprazolam is involved, along with the methods for its determination in biological samples are presented.

\section{Chemistry}

Flualprazolam is a fluorinated triazolobenzodiazepine, containing a fluorine atom in the ortho-position of the phenyl ring of alprazolam (Fig. 1). The IUPAC name of flualprazolam is 8-chloro-6-(2-fluorophenyl)-1-methyl-4H-[1,2,4] triazolo[4,3-a][1,4]benzodiazepine and its CAS number is 28910-91-0 [9]. Flualprazolam has the molecular formula of $\mathrm{C}_{17} \mathrm{H}_{12} \mathrm{ClFN}_{4}$, a molecular weight of $326.75 \mathrm{~g} / \mathrm{mol}$ and a melting point of $203-204{ }^{\circ} \mathrm{C}$ [4]. It is an off-white powder, soluble in dichloromethane and methanol and partially soluble in water [10]. Flualprazolam shares a similar structure with other controlled benzodiazepines, such as alprazolam and triazolam as well as other novel benzodiazepines, such as flubromazolam (Fig. 1) [11].

\section{Synthesis}

Flualprazolam was synthesized in the 1960s and its synthesis was patented in 1976 by Hester, but was never marketed as a medicinal drug as described above. Its synthesis started by refluxing a solution of 7-chloro-1,3-dihydro-5(o-fluorophenyl)-2H-1,4-benzodiazepine-2-thione and acetic acid hydrazide in 1-butanol for $12 \mathrm{~h}$ while bubbling a stream of nitrogen through the reaction mixture. The solvent was evaporated in vacuo. The residue was treated with water and extracted with dichloromethane. The organic layer was dried over anhydrous sodium sulfate and concentrated to an oily residue. Flualprazolam was produced through crystallization from the crude oily residue after treatment with a mixture of ethyl acetate/hexane [4].

An alternative synthesis of flualprazolam using a 1,4-benzodiazepine $N$-nitrosoamidine as precursor has been described by Fustero et al. [12]. $N$-nitrosoamidine was treated with acetylhydrazine $\left(\mathrm{CH}_{3} \mathrm{CONHNH}_{2}\right)$ forming the corresponding amidine, which was then cyclized in dimethylformamide at $120{ }^{\circ} \mathrm{C}$ for $1 \mathrm{~h}$ with the presence of $p$-toluenesulfonic acid (TsOH). The reaction was quenched with saturated sodium bicarbonate after cooling at room temperature. The reaction mixture was extracted with dichloromethane and the combined organic layers were concentrated, dried, and subjected to flash column chromatography, obtaining flualprazolam as final product (Fig. 2).

\section{Pharmacology and toxicology}

There are limited published data concerning pharmacology and toxicology of flualprazolam. Due to its chemical structure, flualprazolam is expected to have similar pharmacokinetic properties with the structurally similar 1,4-triazolobenzodiazepines alprazolam and triazolam, presenting rapid absorption and high binding affinity to plasma proteins [13].

The metabolism of flualprazolam has been investigated in vitro using pooled human liver microsomes (pHLM). One dihydroxylated and two monohydroxylated metabolites were formed through the hydroxylation at $\mathrm{C}_{4}$ of the diazepine core and/or at $\alpha-\mathrm{C}$ atom of the triazolo ring [14]. In another study [15], flualprazolam incubations with pHLS9 and HepaRG cells were used to study the metabolic pattern of flualprazolam. In total, six metabolites were detected, three derived through phase I and three through<smiles>Cc1nnc2n1-c1ccc(Cl)cc1C(c1ccccc1F)=NC2</smiles>

Flualprazolam<smiles>Cc1nnc2n1-c1ccc(Cl)cc1C(c1ccccc1)=NC2</smiles>

Alprazolam<smiles>Cc1nnc2n1-c1ccc(Cl)cc1C(c1ccccc1Cl)=NC2</smiles>

Triazolam<smiles>Cc1nnc2n1-c1ccc(Br)cc1C(c1ccccc1F)=NC2</smiles>

Flubromazolam

Fig. 1 Chemical structure of flualprazolam, alprazolam, triazolam and flubromazolam 
phase II reactions. An additional phase II metabolite was observed in a urine sample of an intoxicated patient. Phase I reactions included mono- and dihydroxylation, and $\alpha$-hydroxyflualprazolam, 4-hydroxyflualprazolam and $\alpha, 4$-dihydroxyflualprazolam were identified (Fig. 3) [15]. Sofalvi et al. [16] consider $\alpha$-hydroxyflualprazolam as the major metabolite of flualprazolam. Single enzyme incubations were used to elucidate the participation of different CYP isoenzymes in these reactions. All three metabolites were detected only in the incubation with CYP3A4, while only the two monohydroxylated metabolites were detected in incubations with CYP2B6, CYP2C19 and CYP3A5, suggesting that CYP3A4 is primarily involved in phase I reactions. Phase II metabolites included glucuronides of the two monohydroxylated metabolites and the glucuronide of the parent drug. The 7th metabolite found only in urine sample was the glucuronide of $\alpha$,4-dihydroxyflualprazolam (Fig. 4). Single enzyme incubations using different uridine 5'-diphospho-glucuronosyltransferase (UGT) enzymes, showed that UGT1A4, UGT2B10 and UGT2B4 catalyze the formation of glucuronides. The first two are involved in $\mathrm{N}$-glucuronidation of flualprazolam and the last one catalyzes the formation of $\alpha$-hydroxyflualprazolam glucuronide. In a plasma sample of a real case, only two ( $\alpha$-hydroxyflualprazolam and 4-hydroxyflualprazolam) out of the 7 metabolites and flualprazolam were detected, while in the respective urine sample, all seven metabolites and the parent compound were found. Due to their higher abundance in urine samples, the $\mathrm{N}$-glucuronide of flualprazolam and the glucuronides of monohydroxylated
Fig. 2 Synthesis of flualprazolam (as proposed by Fustero et al. [12])<smiles>CC(N=O)C1=Nc2ccc(Cl)cc2C(c2ccccc2F)=NC1</smiles>

1,4-Benzodiazepine $N$-nitrosoamidine<smiles>OCc1nnc2n1-c1ccc(Cl)cc1C(c1ccccc1F)=NC2</smiles>

$\alpha$-Hydroxyflualprazolam<smiles></smiles>

$\alpha$-Hydroxyflualprazolam glucuronide<smiles>Cc1nnc2n1-c1ccc(Cl)cc1C(c1ccccc1F)=NC2O</smiles>

4-Hydroxyflualprazolam<smiles>Cc1nnc2n1-c1ccc(Cl)cc1C(c1ccccc1F)=NC2O</smiles>

4-Hydroxyflualprazolam glucuronide<smiles>Cc1nnc2n1-c1ccc(Cl)cc1C(c1ccccc1F)=NC2</smiles>

Flualprazolam<smiles>Cc1nnc2n1-c1ccc(Cl)cc1C(c1ccccc1F)=NC2</smiles>

Flualprazolam glucuronide

Fig. 3 Phase I metabolites of flualprazolam

Fig. 4 Phase II metabolites of flualprazolam

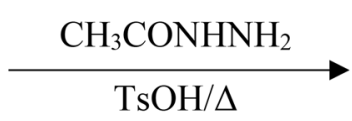


metabolites are suggested to be used as screening targets for the toxicological analysis of urine samples [15]. There are no published data concerning the activities of flualprazolam metabolites.

Although the pharmacological properties of flualprazolam have not extensively been studied, it is believed that flualprazolam acts as a positive allosteric modulator at $\mathrm{GABA}_{\mathrm{A}}$ receptors, as it has a typical benzodiazepine core structure, thus presenting anxiolytic, sedative and hypnotic effects [17]. Preclinical studies described in the patent application indicated that flualprazolam demonstrated sedative effects on the chimney test with intraperitoneal (i.p.) doses of $0.056 \mathrm{mg} / \mathrm{kg}$; and a sedative dose in the dish test of $0.016 \mathrm{mg} / \mathrm{kg}$ with i.p. administration. Sedative/tranquilizer effects were also seen on the pedestal test at i.p. doses of $0.028 \mathrm{mg} / \mathrm{kg}$ [4].

Tests using mice models have been conducted to evaluate the comparative potency of substituted benzodiazepines including flualprazolam. Results indicated that flualprazolam was similarly potent to triazolam and more potent than alprazolam [18]. Reports from drug addicts confirmed the above finding [19]. The observed increase in the potency of flualprazolam as compared to alprazolam could be attributed to the substitution of fluorine in the ortho-position of the phenyl ring of alprazolam molecule, since fluorination of a drug molecule has been shown to increase binding affinity to the receptors and potency [20,21].

The onset of action of flualprazolam, when administered orally, is reported to be $10-30 \mathrm{~min}$, and the duration of action ranges between 6 and $14 \mathrm{~h}$, exhibiting longer duration than alprazolam. Reported doses of flualprazolam range from 0.125 to $2 \mathrm{mg}$ [22], while common doses range from 0.25 to $0.5 \mathrm{mg}$ [23]. Benzodiazepine addicts report higher starting doses, starting from $0.75 \mathrm{mg}$ [19].

According to online users' forums, flualprazolam is mainly used as hypnotic/sedative than as anxiolytic, causing 'relaxation and immediate feelings of sleepiness'. A more intense hangover feeling as compared to other benzodiazepines has been described. Its higher potency has been observed by users, who suggest that flualprazolam is $3-4$ times stronger than alprazolam and equivalent to clonazepam. A user has claimed that 'It's the most dangerous worst benzo I've ever tried...the highest I've gone is $1 \mathrm{mg}$ and that puts me to sleep with no memory of falling asleep... The euphoria will last a few hours, but the main benzodiazepine effects will last well into the next day if taken in doses of 0.75-2 mg'. 'Tolerance seems to build quickly', and withdrawal symptoms are mainly consisting of 'tinnitus and insomnia while I actually was in the drugged sleep. Then I had one very horrible week. I could not sleep for 3 days... Also had extreme memory problems for a week after I stopped using them'. Moreover, disinhibition and criminal activity have been observed [19, 24].
There are no published studies concerning the evaluation of the toxicity of flualprazolam in animals or humans; however, the drug has been associated with numerous drug user deaths and cases of clinical intoxications [9, 25]. Observations show that flualprazolam, when used in combination with other CNS depressants such as opioids or alcohol, can cause serious physical and psychological harm. High doses, especially in combination with opioids, may cause prolonged severe sedation, loss of consciousness, breathing difficulties, severe respiratory depression associated with coma or even death, as the opioid-induced depression is potentiated by flualprazolam [7-9, 25]. The fact that fatal overdoses involving flualprazolam are normally poly-substance cases makes understanding its potential to cause harm difficult [2].

\section{Prevalence and use}

Like other NPS, flualprazolam is widely distributed through the Internet in the illicit drug market, labeled as 'research chemical' and/or 'not for human consumption' and under the street name of 'Flualp' and 'Hulk' [8]. Flualprazolam is mostly sold as counterfeit tablets marked as Xanax/Alprazolam, with an identical appearance and labeling to the commercially available tablets. It is also sold as Xanax bars, alone or in combination with other NPS benzodiazepines, such as etizolam, adinazolam or other unidentified substances. Pellets, blotters and a liquid form of flualprazolam also exist in the market, while powders containing flualprazolam also contain other harmful substances, such as fentanyl, cocaine or MDMA $[11,26]$. A mixture of flualprazolam with diphenhydramine, quinine, fentanyl and cocaine is sold as a powder under the name 'Dope' [26]. Flualprazolam has also been used as adulterant to isotonitazene, a new synthetic opioid [27].

The abuse of flualprazolam is often combined with that of other psychoactive substances as described above. Toxicological analysis of biological samples of flualprazolam users showed that prescribed drugs such as buprenorphine, or other opioids, as well as pregabalin and gabapentin were also detected [28]. The concurrent use of flualprazolam with other NPS benzodiazepines, such as flubromazolam and phenazepam has also been reported [29].

The first report about flualprazolam concerned its identification in the liquid of two seized bottles used to anesthetize the victim of a robbery in 2017 in China [5]. In the next years, the use of flualprazolam has expanded rapidly. According to the National Forensic Laboratory Information System (NFLIS), the recorded number of flualprazolamrelated cases in USA increased significantly, from one in 2017, to 112 in 2018 and to 1624 in 2019. The National Poison Data System of American Association of Poison Control Centers (AAPCC) reported two flualprazolam intoxications 
in 2018 and 11 in 2019, where flualprazolam was the only drug involved [8]. According to the annual Emerging Threat Reports of the Special Testing and Research Laboratory of Drug Enforcement Administration (DEA) for 2018 and 2019, flualprazolam was identified in 2 and 31 seizures, respectively [30, 31]. In the respective report for mid-2020, flualprazolam was reported in 36 seizures accounting for $47 \%$ of total NPS benzodiazepine identifications [32]. In California USA, 123 DUID cases positive for flualprazolam were reported between May 2018 and August 2019, while NMS Labs in Pennsylvania reported 32 DUID cases from 13 different states, between June and November 2019, where flualprazolam was confirmed [25]. As many as 197 postmortem and DUID cases collected in the period between August 2019 and February 2020 from 28 different USA states and one province of Canada, were found positive for flualprazolam. The highest number of positive cases $(n=44)$ was found in Illinois, while four states (Indiana, Michigan, California and Tennessee) each had more than ten positive cases [21]. In two published Trend Reports, the Center for Forensic Science Research and Education (CFSRE) highlighted the detection of 29 and 63 flualprazolam-related cases for the first two trimesters of 2020, respectively. The frequent use of flualprazolam in combination with fentanyl was noticed in both reports [33, 34].

In Europe, flualprazolam was first seized in Sweden in November 2017, while in Finland in February 2018. Kriikku et al. [28] reported 33 postmortem and DUID cases, in Sweden and Finland, where flualprazolam was involved, until December 2019. According to European Monitoring Centre for Drugs and Drug Addiction (EMCDDA) publishing a report on flualprazolam in March 2019, flualprazolam had been seized in Denmark, Finland, Germany, Slovenia, Sweden and Norway mainly as tablets [35].

Flualprazolam was one of the two new NPS benzodiazepines, along with flunitrazolam, seized in the UK from 2017 to July 2019. According to the National Crime Agency (NCA), 17 seizures of flualprazolam had been reported during that period [35]. In the reports of Welsh Emerging Drug \& Identification of Novel Substances (WEDINOS), from July to September 2019, flualprazolam was the 8th most commonly identified substance [36], while in the following trimester, it gained more interest and ranked as the 5th most commonly detected substance [37]. In their annual report for 2019-2020, flualprazolam was among the ten most common substances identified [38]. The number of identifications of flualprazolam in counterfeit tablets was almost doubled from 17 in the third trimester of 2019, to 30 in the fourth trimester of the same year. In the UK, flualprazolam has been associated with 12 deaths, as a contributing factor, until March 2020 [35].

Flualprazolam was found in 33 postmortem cases reported to UNODC from January 2019 to April 2020, where it was assessed to have either contributed to or been the cause of death. The presence of NPS benzodiazepines was identified in a large proportion that was reported to UNODC DUID cases, with flualprazolam and flubromazolam representing the two most reported NPS at 51 and $22 \%$, respectively [2].

There are also more cases where the use of flualprazolam has been reported [39], but these cases have not been published in the scientific literature.

\section{Intoxications and fatal cases related to flualprazolam}

The expanding abuse of flualprazolam worldwide has resulted in numerous cases associated to flualprazolam in a few years. The number of forensic cases, where flualprazolam is involved, is continuously increasing, along with the number of drivers under the influence of flualprazolam. Cases of clinical intoxications have also been reported in the literature [11].

Flualprazolam has been identified in numerous postmortem blood samples collected from drug addicts [3, 21, 28, 40]. The analysis of 171 samples found positive for flualprazolam in USA was conducted by Papsun et al. [21], between August 2019 and February 2020. Most of the decedents were male with a median age of 34 years. The concentration range of flualprazolam in blood samples was $2.0-620 \mathrm{ng} / \mathrm{mL}$ with a median concentration of $8.2 \mathrm{ng} / \mathrm{mL}$. The concurrent use of other common psychoactive substances such as opioids, and mainly fentanyl was observed. Other findings included isotonitazene and other NPS benzodiazepines such as etizolam. As many as 23 flualprazolam-related deaths were recorded in Sweden and Finland until December 2019, most of which were due to fatal poisoning. Most of the users were male and their median age was 30 years. The flualprazolam concentration range in blood samples was $3.0-68.0 \mathrm{ng} / \mathrm{g}$, with a median concentration of $18.0 \mathrm{ng} / \mathrm{g}$. Both medicinal and illegal drugs were also detected in blood samples, with buprenorphine being the most common finding, followed by alcohol, pregabalin and gabapentin, or psychoactive substances such as amphetamine and morphine [28]. A single femoral blood concentration of $1.94 \mathrm{ng} / \mathrm{mL}$ has also been reported by Mei et al. [40], when a newly developed method including flualprazolam was applied for the investigation of forensic cases. In nine flualprazolam-related cases that were investigated in UK between April and December 2019, flualprazolam found was considered to be a contributing factor and not the compound responsible for these deaths, as numerous other drugs, such as heroin metabolites, cocaine and other NPS, were detected [3].

Blumenberg et al. [11] described the first confirmed clinical flualprazolam case where six teenagers consumed flualprazolam, after receiving the drug as a free sample, believing 
that it was commercial Xanax. Flualprazolam was identified by the analysis of a fragment of a not consumed tablet and it was concluded that the whole tablet contained approximately $2.75-3 \mathrm{mg}$ of flualprazolam. Four patients displayed the expected symptoms of a benzodiazepine intoxication such as lethargy, slurred speech and confusion, while the other two were asymptomatic. One patient developed mild respiratory depression and the concentration of flualprazolam in its serum was $14.6 \mathrm{ng} / \mathrm{mL}$. As stated in the relative article, this concentration is within the therapeutic range of alprazolam. In three patients, flualprazolam was detected in their urine samples. All symptoms reported subsided within $6 \mathrm{~h}$ [11].

In California, USA between May 2018 and August 2019, 123 cases of flualprazolam were reported during the investigation of DUID cases. The flualprazolam concentrations ranged from 5 to $154 \mathrm{ng} / \mathrm{mL}$, with a median concentration of $18 \mathrm{ng} / \mathrm{mL}$. NMS Labs in Horsham, Pennsylvania confirmed 12 DUID cases, between June 2019 and November 2019 , positive for flualprazolam. The concentrations of flualprazolam reported in these cases ranged from 8.3 to $68 \mathrm{ng} /$ $\mathrm{mL}$, while the median concentration was $12 \mathrm{ng} / \mathrm{mL}$ [25]. Flualprazolam has been also detected in 22 blood samples collected during the investigation of DUID cases from four USA states (Pennsylvania, Mississippi, Texas, and Oregon) between August 2019 and February 2020. The average age of the drivers was 27 years and the majority of them were male. The flualprazolam concentration range was found to be between 4.4 and $68 \mathrm{ng} / \mathrm{mL}$. In five cases, opioids, such as methadone and buprenorphine were also present. In one case, where flualprazolam was the only compound detected, blood concentration was $13 \mathrm{ng} / \mathrm{mL}$ and the driver displayed incoordination, delayed comprehension and reaction time, and lethargic behavior [21]. The relationship between blood concentrations of flualprazolam and impairment of driving ability was also evaluated by Heide et al. [29]. In ten cases positive for flualprazolam, the measured concentrations ranged between 3.3 and $56 \mathrm{ng} / \mathrm{mL}$, with a median concentration of $8 \mathrm{ng} / \mathrm{mL}$. The results indicated that flualprazolam contributes to impairment, as in a mono-substance case, the concentration found was $15 \mathrm{ng} / \mathrm{mL}$ and the person's driving ability was described as 'considerably impaired'. In two other DUID cases reported by Garcia et al. [41], flualprazolam was detected at concentrations of 5.4 and $45 \mathrm{ng} / \mathrm{mL}$, respectively. Cannabis and/or ethanol were also consumed by the drivers.

\section{Analysis of flualprazolam in biological samples}

Many of NPS benzodiazepines give positive results to commercial urine immunoassays [42]. Flualprazolam has been shown to cross-react with commercially available urine benzodiazepine immunoassay kits, such as immunochromatographic tests (cutoff $150 \mathrm{ng} / \mathrm{mL}$ of nordiazepam) [11] or immunochemical assays (enzyme-linked immunosorbent assay, ELISA) (cutoff $10 \mathrm{ng} / \mathrm{mL}$ of clonazepam) [41]. The cross-reactivity of flualprazolam to benzodiazepine-specific lateral flow immunoassay test strips that were designed to detect alprazolam in drug samples has also been evaluated. Positive results were given when concentrations of flualprazolam in water drug solutions were higher than $0.5 \mu \mathrm{g} / \mathrm{mL}$ [43].

The isolation of flualprazolam from blood or urine samples has been performed by liquid-liquid extraction with butyl acetate [28], a mixture of methyl tert-butyl ether/1-chlorobutane (60:40, v/v) [21], ethyl acetate/heptane $(4: 1, \mathrm{v} / \mathrm{v})$ or with methyl tert-butyl ether [29] after alkalinization. For the solid-phase extraction (SPE) of flualprazolam, mixed-mode (strong cation exchange and hydrophobic) [16] and Preconditioned Clean Screen XCEL I columns [40] have been used. Protein precipitation with acetonitrile or different acetonitrile/methanol mixtures has been used in some methods, prior to SPE [16] or before the injection of sample into the liquid chromatography (LC) system $[11,41]$. When urine samples were analyzed, their incubation with $\beta$-glucuronidase enzyme preceded extraction $[11,16]$.

Several chromatographic methods for the identification and quantification of flualprazolam in biological samples have been described. There is only one published method using gas chromatography-mass spectrometry (GC-MS). A ZB-DRUG-1 capillary column was used for the chromatographic separation of flualprazolam from other benzodiazepines and their metabolites. Gas chromatograph was coupled to a negative chemical ionization (NCI)-MS detector. GC-NCI-MS analysis was operated in selected ion monitoring (SIM) mode and the ions $m / z 326$ and 328 were selected for the identification of flualprazolam [28].

Most of the published methods use LC coupled to tandem mass spectrometry (MS/MS) [3, 16, 21, 29, 40, 41] or quadrupole time-of-flight (QTOF)-MS [11]. Both techniques have also been used for the screening of blood or urine samples [3, 21, 29]. For the chromatographic separation, C18 [3, 11, 21, 40], biphenyl [41] and phenyl-hexyl (Ph6) $[16,29]$ columns have been used.

Analytical data, including electron ionization (EI), MS/ MS and QTOF-MS spectra of flualprazolam are available [44]. ${ }^{13} \mathrm{C}$ and ${ }^{1} \mathrm{H}$ nuclear magnetic resonance (NMR), Fourier transform infrared (FTIR)-attenuated total reflectance (ATR) and infrared (IR) spectra are also given in the literature $[5,10]$. These data could be used from toxicology laboratories that may encounter this emerging benzodiazepine in caseworks. 


\section{Legal status}

Flualprazolam is largely not scheduled worldwide, although in some European countries and some USA states, it is considered a controlled substance. In Europe, due to different legislations, it is classified in different Schedules of the local drug laws. In Sweden, flualprazolam has been added in Annex 1 of the amended Ordinance (1992:1554) on the control of narcotic drugs, since 2018 [45]. In Finland, it has been classified as a narcotic and placed in Schedule IV of the Single Convention on Narcotic Drugs of 1961, after a government decree in July 2019 [46]. In the UK, following a recommendation of the Advisory Council on the Misuse of Drugs (ACMD) made in April 2020, flualprazolam became controlled from September 2020 as a Class C drug under Misuse of Drugs Act 1971 and placed in Schedule 1 to the Misuse of Drug Regulations 2001 [7].

In USA, flualprazolam is not currently controlled under the Controlled Substance Act but is controlled in a number of states, such as Louisiana and Virginia [8, 47, 48]. In Oregon, it is placed under Schedule 1 as a substituted benzodiazepine and according to the state legislation [49], while in Hawaii, its scheduling will take effect on the 15th of November 2020 [48].

According to the Office of Drug Control of the Australian Government, the import and export of flualprazolam is controlled [50].

In October 2019, the World Health Organization's Expert Committee on Drug Dependence (WHO ECDD) published a Critical Review Report about flualprazolam [9]. WHO Technical Report No 1026, based on the potential of flualprazolam to produce dependence and CNS depression, as other controlled benzodiazepines, and existing evidence that it can be a public health threat and a social problem, recommended that flualprazolam should be added to Schedule IV of the 1971 Convention on Psychotropic Substances [51]. The United Nations Commission on Narcotic Drugs (CND) adopted the WHO ECDD recommendation at their 63rd Session held in March 2020. The addition of flualprazolam to Schedule IV of the 1971 Convention on Psychotropic Substances is decided to force on the 3rd of November 2020, to avoid an imminent hazard to the health and safety of the public [52].

In Japan, flualprazolam was designated as a narcotic since July 2020 [53].

\section{Conclusions}

Flualprazolam, a designer benzodiazepine that has recently emerged into the illicit drug market has been involved in an increasing number of forensic as well as clinical cases. Despite the lack of scientific information on the clinical effects and pharmacologic characteristics of flualprazolam, this new NPS has similar pharmacological action but much stronger effects than alprazolam, also confirmed by anecdotal online reports. Symptoms of flualprazolam intoxication include sedation, hypnotic effects and amnesia, while its combined use with other CNS psychoactive depressants increases the risk of respiratory depression and death. The urgent international control of flualprazolam, aiming to reduce prevalence and to prevent its increasing abuse worldwide, is necessary, and it has been proposed and adopted by international stakeholders.

\section{Compliance with ethical standards}

Conflict of interest There are no financial or other relations that could lead to a conflict of interest.

Ethical approval The article does not contain any studies with human participants or animals performed by any of the authors.

Open Access This article is licensed under a Creative Commons Attribution 4.0 International License, which permits use, sharing, adaptation, distribution and reproduction in any medium or format, as long as you give appropriate credit to the original author(s) and the source, provide a link to the Creative Commons licence, and indicate if changes were made. The images or other third party material in this article are included in the article's Creative Commons licence, unless indicated otherwise in a credit line to the material. If material is not included in the article's Creative Commons licence and your intended use is not permitted by statutory regulation or exceeds the permitted use, you will need to obtain permission directly from the copyright holder. To view a copy of this licence, visit http://creativecommons.org/licenses/by/4.0/.

\section{References}

1. Manchester KR, Lomas EC, Waters L, Dempsey FC, Maskell PD (2018) The emergence of new psychoactive substance (NPS) benzodiazepines: a review. Drug Test Anal 10:37-53. https://doi. org/10.1002/dta.2211

2. United Nations Office on Drugs and Crime UNODC (2020) Current NPS threats - volume III. https://www.unodc.org/documents/ scientific/Current_NPS_Threats_Vol.3.pdf. Accessed 22 Oct 2020

3. Rice K, Hikin L, Lawson A, Smith PR, Morley S (2020) Quantification of flualprazolam in blood by LC-MS-MS: a case series of nine deaths. J Anal Toxicol. https://doi.org/10.1093/jat/bkaa0 98

4. Hester JB Jr (1976) United States patent "US3987052A: 6-phenyl-4H-s-triazolo[4,3-a][1,4]benzodiazepines." https://paten ts.google.com/patent/US3987052A/en. Accessed 21 Oct 2020 
5. Qian Z, Liu C, Huang J, Deng Q, Hua Z (2020) Identification of the designer benzodiazepine 8-chloro-6-(2-fluorophenyl)-1-methyl-4H-[1,2,4]triazolo[4,3-a][1,4]benzodiazepine (flualprazolam) in an anesthesia robbery case. Forensic Toxicol 38:269-276. https ://doi.org/10.1007/s11419-019-00501-1

6. The Center for Forensic Science Research and Education (2019) Flualprazolam: potent benzodiazepine identified among death and impaired driving cases in the U.S. https://www.npsdiscovery.org/ wp-content/uploads/2019/12/Public-Alert_Flualprazolam_NPSDiscovery_120519.pdf. Accessed 22 Oct 2020

7. Home Office on GOV.UK (2020) Three new benzodiazepines to become Class C drugs. https://www.gov.uk/government/news/ three-new-benzodiazepines-to-become-class-c-drugs. Accessed 21 Oct 2020

8. Drug Enforcement Administation, Diversion Control Division, Drug and Chemical Evaluation Section (2020) Flualprazolam https://www.deadiversion.usdoj.gov/drug_chem_info/flualp.pdf. Accessed 21 Oct 2020

9. WHO Expert Committee on Drug Dependence (2019) Critical review report: flualprazolam https://www.who.int/medic ines/access/controlled-substances/Final_Flualprazolam.pdf. Accessed 22 Oct 2020

10. National Forensic Laboratory of Slovenia (2018) Analytical report: flualprazolam. https://www.policija.si/apps/nfl_ response_web/0_Analytical_Reports_final/FlualprazolamID-1903-18_report.pdf. Accessed 22 Oct 2020

11. Blumenberg A, Hughes A, Reckers A, Ellison R, Gerona R (2020) Flualprazolam: report of an outbreak of a new psychoactive substance in adolescents. Pediatrics 146:e20192953. https ://doi.org/10.1542/peds.2019-2953 (open access article)

12. Fustero S, González J, del Pozo C (2006) 1,4-Benzodiazepine $N$-nitrosoamidines: useful intermediates in the synthesis of tricyclic benzodiazepines. Molecules 11:583-588. https://doi. org/10.3390/11080583 (open access article)

13. Garzone PD, Kroboth PD (1989) Pharmacokinetics of the newer benzodiazepines. Clin Pharmacokinet 16:337-364. https://doi. org/10.2165/00003088-198916060-00002

14. Wilde M, Sommer M, Auwärter V (2018) Identification and characterization of the new designer benzodiazepines bromazolam, flualprazolam and fluclotizolam. TIAFT 2018 Poster P167, Ghent 28 Aug 2018

15. Wagmann L, Manier SK, Bambauer TP, Felske C, Eckstein N, Flockerzi V, Meyer MR (2020) Toxicokinetics and analytical toxicology of flualprazolam: metabolic fate, isozyme mapping, human plasma concentration and main urinary excretion products. J Anal Toxicol 44:549-558. https://doi.org/10.1093/jat/ bkaa019

16. Sofalvi S, Lavins ES, Kaspar CK, Michel HM, Mitchell-Mata CL, Huestis MA, Apollonio LG (2020) Development and validation of an LC-MS-MS method for the detection of 40 benzodiazepines and three Z-drugs in blood and urine by solid-phase extraction. $\mathrm{J}$ Anal Toxicol 44:708-717. https://doi.org/10.1093/jat/bkaa072

17. Sigel E, Ernst M (2018) The benzodiazepine binding sites of $\mathrm{GABA}_{\mathrm{A}}$ receptors. Trends Pharmacol Sci 39:659-671. https:// doi.org/10.1016/j.tips.2018.03.006

18. Hester JB Jr, Rudzik AD, Kamdar BV (1971) 6-Phenyl-4H-striazolo[4,3- $\alpha][1,4]$ benzodiazepines which have central nervous system depressant activity. J Med Chem 14:1078-1081. https:// doi.org/10.1021/jm00293a015

19. Reddit (2019) Flualprazolam vs alprazolam Reddit sinemet. http:// wp.labo-style.com/forum/archive.php?id=b93ba2-flualprazolamvs-alprazolam-reddit-sinemet. Accessed 21 Oct 2020

20. Gillis EP, Eastman KJ, Hill MD, Donnelly DJ, Meanwell NA (2015) Applications of fluorine in medicinal chemistry. J Med Chem 58:8315-8359. https://doi.org/10.1021/acs.jmedc hem. 5 b00258
21. Papsun DM, Krotulski AJ, Homan J, Temporal KDH, Logan BK (2020) Flualprazolam blood concentrations in 197 forensic investigation cases. J Anal Toxicol. https://doi.org/10.1093/jat/bkaa070

22. Zawilska JB, Wojcieszak J (2019) An expanding world of new psychoactive substances-designer benzodiazepines. Neurotoxicology 73:8-16. https://doi.org/10.1016/j.neuro.2019.02.015

23. Moosman B, Auwärter V (2018) Designer benzodiazepines: another class of new psychoactive substances. Handb Exp Pharmacol 252:383-410. https://doi.org/10.1007/164_2018_154

24. Erowid (1995-2020) Erowid experience vault. https://erowid.org/ experiences/. Accessed 21 Oct 2020

25. Papsun D, Triebold C (2020) Emerging drug: flualprazolam. Toxtalk 43:14-16. https://www.soft-tox.org/files/toxtalk/ToxTalk\%20 Dec_2019_FINAL.pdf. Accessed 21 Oct 2020

26. DrugsData (2020) Erowid's anonymous drug analysis program formely called EcstasyData. https://www.drugsdata.org. Accessed 21 Oct 2020

27. The Center of Forensic Science Research and Education (2020) The rise of brorphine - a potent new synthetic opioid identified in the Midwestern United States. https://www.npsdiscovery.org/ the-rise-of-brorphine-a-potent-new-synthetic-opioid-identified -in-the-midwestern-united-states/. Accessed 22 Oct 2020

28. Kriikku P, Rasanen I, Ojanperä I, Thelander G, Kronstrand R, Vikingsson S (2020) Femoral blood concentrations of flualprazolam in 33 postmortem cases. Forensic Sci Int 307:110101. https ://doi.org/10.1016/j.forsciint.2019.110101

29. Heide G, Høiseth G, Middelkoop G, Øiestad ÅML (2020) Blood concentrations of designer benzodiazepines: relation to impairment and findings in forensic cases. J Anal Toxicol. https://doi. org/10.1093/jat/bkaa043 (open access article)

30. Drug Enforcement Administration Special Testing and Research Laboratory (2018) Emerging threat report, annual 2018. https:// cesar.umd.edu/sites/cesar.umd.edu/files/pubs/DEA-EmergingThreat-Report-2018-Annual.pdf. Accessed 21 Oct 2020

31. Drug Enforcement Administration Special Testing and Research Laboratory (2019) Emerging threat report, annual 2019. https:// cesar.umd.edu/sites/cesar.umd.edu/files/pubs/DEA-EmergingThreat-Report-2019-Annual.pdf. Accessed 21 Oct 2020

32. Drug Enforcement Administration Special Testing and Research Laboratory (2020) Emerging threat report, mid - year 2020. https ://cesar.umd.edu/sites/cesar.umd.edu/files/dea-emerging-threa t-report-2020-mid-year.pdf. Accessed 21 Oct 2020

33. The Center for Forensic Science Research and Education (2020) Trend report: Q1 2020, NPS benzodiazepines in the United States. https://www.npsdiscovery.org/wp-content/uploads/2020/04/2020Q1_NPS-Benzodiazepines_Trend-Report.pdf. Accessed 21 Oct 2020

34. The Center for Forensic Science Research and Education (2020) Trend report: Q2 2020 NPS benzodiazepines in the United States. https://www.npsdiscovery.org/wp-content/uploads/2020/07/2020Q2_NPS-Benzodiazepines_Trend-Report_rev.pdf. Accessed 21 Oct 2020

35. Advisory Council on the Misuse of Drugs (2020) A review of the evidence of use and harms of novel benzodiazepines. https ://assets.publishing.service.gov.uk/government/uploads/system/ uploads/attachment_data/file/881969/ACMD_report_-_a_revie w_of_the_evidence_of_use_and_harms_of_novel_benzodiaze pines.pdf. Accessed 21 Oct 2020

36. Wedinos.org (2019) Issue 17, benzodiazepines. https://www. wedinos.org/resources/downloads/Philtre_Issue_17.pdf. Accessed 22 Oct 2020

37. Wedinos.org (2019) Issue 18, benzodiazepines. https://www. wedinos.org/resources/downloads/Philtre_Issue_18.pdf. Accessed 22 Oct 2020 
38. Wedinos.org (2020) Annual report 2019-2020. https://phw. nhs.wales/publications/publications1/wedinos-annual-report/. Accessed 22 Oct 2020

39. Mirror, UK (2020) https://www.mirror.co.uk. Accessed 21 Oct 2020

40. Mei V, Concheiro M, Pardi J, Cooper G (2019) Validation of an LC-MS/MS method for the quantification of 13 designer benzodiazepines in blood. J Anal Toxicol 43:688-695. https://doi. org/10.1093/jat/bkz063

41. Garcia L, Tiscione NB, Yeatman DT, Richards-Waugh L (2020) Novel and non-routine benzodiazepines and suvorexant by LCMS-MS. J Anal Toxicol. https://doi.org/10.1093/jat/bkaa109

42. Pettersson Bergstrand M, Helander A, Hansson T, Beck O (2017) Detectability of designer benzodiazepines in CEDIA, EMIT II Plus, HEIA, and KIMS II immunochemical screening assays. Drug Test Anal 9:640-645. https://doi.org/10.1002/dta.2003

43. British Columbia Centre on Substance Use (2020) Detection of etizolam, flualprazolam, and flubromazolam by benzodiazepinespecific lateral flow immunoassay test strips. https://www.bccsu ca. Accessed 22 Oct 2020

44. The Center for Forensic Science Research and Education (2019) Flualprazolam. https://www.issup.net/files/2019-06/Flualprazo lam_062519_ToxicologyAnalyticalReport.pdf. Accessed 22 Oct 2020

45. The Swedish Prime Minister's Office (2020) Swedish code of statutes. https://www.svenskforfattningssamling.se/english.html. Accessed 22 Oct 2020

46. European Commission (2019) Government Decree amending Annex IV to the Government Decree on substances, preparations and plants to be classified as narcotics The following 7 new substances are classified: adinazolam, flualprazolam, fluclotizolam, flunitrazolam, metizolam, nitrazolam, 3-hydroxyphenazepam as narcotics. https://ec.europa.eu/. Accessed 21 Oct 2020
47. Federal Register (2019) Vol. 84, No. 175, September 10, 2019, Notices p 47527. https://www.govinfo.gov/content/pkg/FR-201909-10/pdf/FR-2019-09-10.pdf. Accessed 22 Oct 2020

48. Department of Public Safety, Narcotics Enforcement Division, State of Hawaii (2020) Emergency controlled substance scheduling action. https://dps.hawaii.gov/wp-content/uploads/2020/10/ notice-of-emergency-Scheduling-Action-etizolam-and-flualprazo lam-notice.pdf. Accessed 22 Oct 2020

49. Oregon Secretary of State (2020) Oregon administrative rules, Board of Pharmacy, chapter 855: division 80 - schedule of controlled substances. https://secure.sos.state.or.us/oard/displayCha pterRules.action?selectedChapter=19. Accessed 22 Oct 2020

50. Australian Government, Office of Drug Control (2020) https:// www.odc.gov.au. Accessed 22 Oct 2020

51. WHO Expert Committee on Drug Dependence (2020) TRS 1026, forty second report. https://www.who.int/publications/i/ item/978-92-4-000184-8. Accessed 22 Oct 2020

52. United Nations Office on Drug and Crime (2020) UNODC resolutions and decisions 2020-2029. https://www.unodc.org/unodc/en/ commissions/CND/Resolutions_Decisions/resolutions-and-decis ions-2020-2029.html. Accessed 21 Oct 2020

53. Ministry of Health, Labour and Welfare of Japan (2020) Ten psychoactive drugs newly designated as narcotics since 9 July 2020. https://www.mhlw.go.jp/stf/houdou/0000212707_00002.html. Accessed 20 Nov 2020 (in Japanese)

Publisher's Note Springer Nature remains neutral with regard to jurisdictional claims in published maps and institutional affiliations. 